\title{
Apoptotic mechanisms in rabbits with blast-induced acute lung injury ${ }^{1}$
}

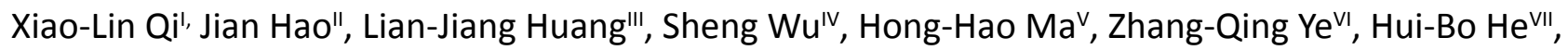
Shu-Wen Li ${ }^{\mathrm{VIII}}$, Cheng-En Li' ${ }^{\mathrm{IX}}$ Xin Huang ${ }^{\mathrm{IX}}$

'Master, Department of Internal Medicine, 123th Hospital of PLA, Pengbu, Anhui Province, China. Acquisition of data. "Master, Chief Physician, Department of Cardiopulmonary Rehabilitation Center, 128th Hangzhou Hospital, Military Hangzhou Clinical College, Anhui Medical University, Hangzhou, Zhejiang Province, China. Analysis of data.

I'Master, Chief Physician, Department of Digestive Internal Medicine, 123th Hospital of PLA, Pengbu, Anhui Province, China. Technical procedures, blood sample determination.

IVMaster, Chief Physician, Department of Cardiopulmonary Rehabilitation Center, 128th Hangzhou Hospital, Military Hangzhou Clinical College of Anhui Medical University, Hangzhou, Zhejiang Province, China. Statistical analysis, collation of experimental samples.

vPhD, Department of Modern Mechanics, University of Science and Technology of China, Hefei, Anhui Province, China. Technical procedures.

"'Master, Department of Digestive Internal Medicine, 123th Hospital of PLA, Pengbu, Anhui Province, China. Bood sample determination.

VIIMaster degree candidate, Department of Heart Internal Medicine, 123th Hospital of PLA, Pengbu, Anhui Province, China. Sample extraction, analysis of data.

VIIIMaster, Chief Physician, Department of Cardiopulmonary Rehabilitation Center, 128th Hangzhou Hospital, Military Hangzhou Clinical College, Anhui Medical University, Hangzhou, Zhejiang Province, China. Sample extraction, analysis of data.

${ }^{\text {x} M a s t e r ~ d e g r e e ~ c a n d i d a t e, ~ D e p a r t m e n t ~ o f ~ C a r d i o p u l m o n a r y ~ R e h a b i l i t a t i o n ~ C e n t e r, ~ 128 t h ~ H a n g z h o u ~ H o s p i t a l, ~ M i l i t a r y ~ H a n g z h o u ~}$ Clinical College, Anhui Medical University, Hangzhou, Zhejiang Province, China. Sample extraction, analysis of data.

\section{Abstract}

Purpose: To investigate the apoptotic mechanisms in rabbits with blast-induced acute lung injury (ALI).

Methods: A total of 40 rabbits were randomly divided into a blank control group $(A, n=10)$ and an experimental group ( $E X P, n=30)$. Explosion-induced chest-ALI models were prepared and sampled at different time points $(4,12$, and $24 \mathrm{~h}$ after modeling, T1-T3) to test the lung dry weight/wet weight ratio (W/D) and arterial oxygen pressure $\left(\mathrm{PaO}_{2}\right)$, apoptosis of lung tissue by the TUNEL assay, and Caspase-3, Bax, and Bcl-2 levels by immunohistochemical analysis. Furthermore, lung tissue was sampled to observe pathological morphology by microscopy.

Results: Under a light microscope, Group EXP exhibited obvious edema in the pulmonary interstitial substance and alveoli, a large number of red blood cells, inflammatory cells, and serous exudation in the alveolar cavity, as well as thickening of the pulmonary interstitial fluid. Compared to Group A, the W/D ratio was significantly increased in Group EXP $(P<0.01)$, while $\mathrm{PaO}_{2}$ was significantly reduced $(P<0.01)$. The apoptosis index was significantly increased $(P<0.01)$, and caspase- 3 and $B a x / B c l-2$ levels were increased $(P<0.01)$.

Conclusion: Apoptosis plays an important role in the occurrence and development of acute lung injury in rabbits by participating in lung injury and promoting the progression of ALI.

Key words: Acute Lung Injury. Apoptosis. Blast injuries. Blood Gas Analysis. Rabbits. 


\section{Introduction}

Acute lung injury (ALI), as a cause of death in explosive blast casualties ${ }^{1}$, is a complex clinical syndrome involving acute inflammation, microvascular damage, and increased pulmonary vascular and epithelial permeability, which results in fatal acute respiratory distress syndrome (ARDS) ${ }^{2}$. ARDS refers to a complex pathological process caused by direct/indirect injury to the body, including assisted ventilation ${ }^{3}$, blood transfusion ${ }^{4}$, inflammation ${ }^{5}$, severe trauma or sepsis ${ }^{6}$, drowning or blast ${ }^{7}$, and even toxins $^{8}$. Currently, the most widely accepted definition of ALI/ARDS is based on the American-European Consensus Conference definition of acute onset respiratory failure with bilateral infiltrates on chest radiograph and pulmonary capillary wedge pressure $<18 \mathrm{mmHg}$ or absence of elevated left atrial pressure ${ }^{9}$, which leads to injury in alveolar epithelial cells and capillary endothelial cells. Pathophysiologically, ALI is characterized by disruption of the alveolar-capillary interface, pro-inflammatory cytokine secretion, and pro-inflammatory cell infiltration ${ }^{10}$. Although many studies and therapeutic trials have been conducted, no effective therapies are available for $A L I$ in the clinic, possibly because its specific mechanism is unclear. The expression of the apoptosis-related proteins $\mathrm{Bax}, \mathrm{Bcl}-2$, and cleaved-caspase- 3 and the ratio of Bax/ $\mathrm{Bcl}-2$ are significantly increased in $\mathrm{ALI}^{11}$. In China, there are more than 20 million ALI/ ARDS cases annually, with a mortality rate of approximately $26.0 \%{ }^{12}$, among which blast-caused ALI/ARDS cases account for a large proportion. ALI pathogeneses are complex, and because of their high mortality, determining the mechanisms can provide a basis for clinical diagnosis and treatment. Recent studies ${ }^{13}$ showed that apoptosis is involved in the pathogenesis of ALI; apoptosis of alveolar epithelial cells and pulmonary capillary endothelial cells in ALI patients is increased and thus the number of cells decreases, promoting disease progression. The aim of this study was to investigate the mechanism of apoptosis in ALI by assessing the levels of plasma caspase-3, Bax, and $\mathrm{Bcl}-2$ in an ALI rabbit model.

\section{Methods}

\section{Animals and grouping}

This study was carried out in strict accordance with the recommendations in the Guide for the Care and Use of Laboratory Animals of the National Institutes of Health. The animal use protocol has been reviewed and approved by the Institutional Animal Care and Use Committee (IACUC) of Anhui Medical University.

A total of 40 healthy New Zealand rabbits (male: female=1:1,2.23 $\pm 0.28 \mathrm{~kg}$ ) were provided by Changlinhe Pharmaceutical Technology Co., Ltd. (Hubei, China, Anhui license number scxk (Wan) 2006-002). The rabbits were divided into a control group ( $A, n$ $=10)$ and an experimental group $(n=30)$; the experimental group was further divided into Groups B-D according to their sampling time (B: 4 h, C: 12 h, and D: $24 h, n=10$ ).

\section{Modeling}

The explosion device developed by the University of Science and Technology of China was used and an appropriate explosive dose and distance were designed according to explosive mechanics-related formula and lung histopathological observation results. Each rabbit was fixed onto a platform and the anterior chest and side chest wall were exposed (other body parts were protected by a self-designed 
protection device). The respiratory rate, heart rate, arterial oxygen pressure $\left(\mathrm{PaO}_{2}\right)$, and wet/ dry lung ratio (W/D) were determined, and the pathological morphology of lung tissue was observed. The rabbits were prepared as an ALI model.

\section{Criteria of $A L I$ model}

$\mathrm{PaO}_{2} / \mathrm{FiO}_{2} \leq 300 \mathrm{mmHg}, \mathrm{W} / \mathrm{D}$, and pathological changes in the lung tissue were used to determine the degree of lung injury and pulmonary edema.

\section{Specimen sampling}

After anesthetization with chloral hydrate $(0.35 \mathrm{~g} / \mathrm{kg})$ via the ear vein, external carotid artery blood was collected from each rabbit for blood gas analysis, as well as the carotid artery blood for centrifugation at $4^{\circ} \mathrm{C}$ and further analysis. Rabbits were sacrificed at the same time, the right lung hilum was ligated, and the left lung was rinsed with $10 \mathrm{~mL}$ of $0.9 \%$ sodium chloride solution (lavage fluid was centrifuged for future use). The middle lobe of the right lung was then dried to detect surface impurities for the dry and wet weights. The right lower lobe was rinsed with $0.9 \%$ sodium chloride and fixed in $10 \%$ formaldehyde. The remaining right lobe was quickly placed in liquid nitrogen for preservation.

\section{Histopathological observation}

The $10 \%$ formaldehyde-fixed right lower lobe was paraffin-embedded, sliced (4-5 $\mu \mathrm{m}$ in thickness), stained with hematoxylin and eosin (HE), and observed by light microscopy.

$W / D$

The middle lobe of the right lung was dried, weighed, and then dried in an $80^{\circ} \mathrm{C}$ oven for $72 \mathrm{~h}$ to determine the dry weight and calculate the W/D.

\section{Arterial blood gas analysis}

Two milliliters of arterial blood were sampled from the disinfected external carotid artery for immediate blood gas analysis. Apoptosis in the lung tissue was detected by the TUNEL method according to the kit instructions; sections were de-waxed in xylene, followed by rehydration in descending concentrations of ethanol, proteinase $\mathrm{K}$ digestion, and enzymatic labeling of free $3^{\prime}-\mathrm{OH}$ DNA termini with digoxigenin-conjugated and unconjugated nucleotide triphosphates by TdT at $37^{\circ} \mathrm{C}$. Labeled apoptotic cells were identified by treating the sections with the peroxidase chromogenic substrate 3,3'-diaminobenzidine (DAB) as recommended by the manufacturer. The cells were blocked and then incubated with primary antibodies $(1: 50,50 \mu \mathrm{L})$, followed by incubation with biotinylated goat anti-mouse IgG and $S A B C$ reagent. For DAB analysis, a positive result is defined as brown nuclei; optical microscopy was performed for counting and analysis of brown chromatin and cytoplasm and analyzed using the grey value. The expression of caspase-3, Bax, and Bcl2 proteins in the lung tissue were detected by a routine SABC method according to the manufacturer's instructions.

\section{Statistical analysis}

Variance analysis and the -q test were conducted, with $\mathrm{P}<0.05$ considered to indicate statistical significance.

\section{Results}

Histopathological observation of lung tissue

Direct observation revealed increased bilateral lung volumes and consolidation with varying degrees of ecchymosis scattered on the surface; partial tissue exhibited red 
consolidation and exudation on its incisal surface. Light microscopy also revealed that the alveolar septum was larger, alveolar cavity was relatively narrowed, and normal alveolar structure was destroyed, together with edema and exudation in the alveolar cavity and pulmonary interstitial substance; some sites exhibited bleeding accompanied by inflammatory cell infiltration (Figure 1).
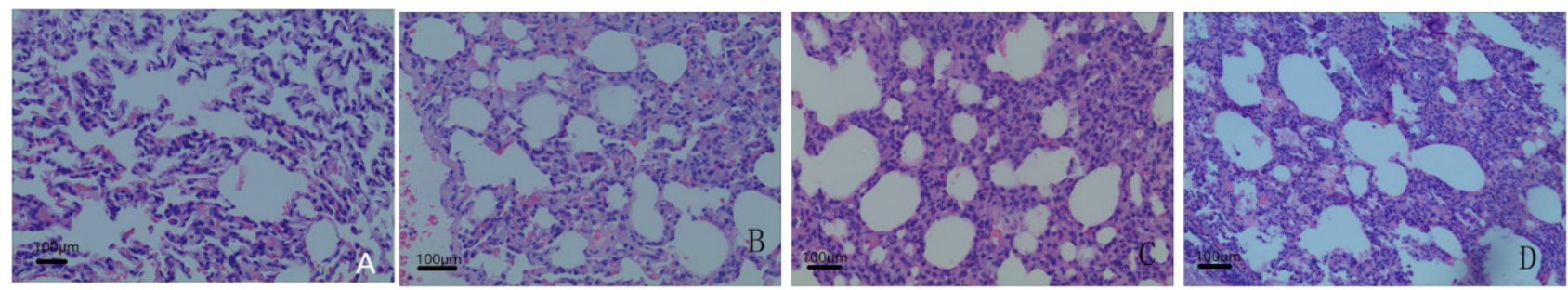

Figure 1 - Histopathological changes in each group (HE ×100). A: Group A; B: Group B; C: Group C; D: Group D.

Comparison of $\mathrm{W} / \mathrm{D}, \mathrm{PaO}_{2}$, and tissue apoptotic index

The apoptotic indices in Groups B-D were significantly higher than in Group $A$
$(\mathrm{P}<0.01)$, while $\mathrm{PaO}_{2}$ was significantly lower than in Group $A(P<0.01)$. The apoptotic indices in Groups $D$ and $C$ were significantly higher than in Group B $(P<0.01)$, while that in Group D was lower than in Group C $(P<0.05)$ (Table 1).

Table 1 - Comparison of W/D, $\mathrm{P}_{a} \mathrm{O}_{2}$, and tissue apoptotic indexes among the 4 groups $(\bar{x} \pm \mathrm{s})$.

\begin{tabular}{lllll} 
Group & $\mathbf{n}$ & $\mathbf{W} / \mathbf{D}$ & $\mathbf{P}_{\mathrm{a}} \mathbf{O}_{\mathbf{2}}(\mathrm{mmHg})$ & Apoptotic index (\%) \\
\hline $\mathrm{A}$ & 10 & $4.63 \pm 0.19$ & $12.65 \pm 0.93$ & $3.02 \pm 0.35$ \\
$\mathrm{~B}$ & 10 & $6.03 \pm 0.26^{* *}$ & $9.49 \pm 1.44^{* *}$ & $14.08 \pm 1.86^{* *}$ \\
$\mathrm{C}$ & 10 & $6.15 \pm 0.23^{* *}$ & $9.08 \pm 1.22^{* *}$ & $22.76 \pm 3.20^{* * \Delta \Delta}$ \\
$\mathrm{D}$ & 10 & $5.83 \pm 0.29^{* * \#}$ & $10.16 \pm 0.93^{* *}$ & $20.69 \pm 1.79^{* * \Delta \Delta}$ \\
$\mathrm{F}$ & - & 81.25 & 19.35 & 185.51 \\
$P$ & - & $<0.01$ & $<0.01$ & $<0.01$ \\
Intra-group MS & - & 0.060 & 1.323 & 4.257 \\
\hline
\end{tabular}

Note: $\mathrm{Q}$ test: compared with group $\mathrm{A},{ }^{* *} P<0.01$; compared with group $\mathrm{B},{ }^{\Delta} P<0.01$; compared with group $\mathrm{C}$, ${ }^{\sharp} P<0.05$.

Comparison of expression of caspase-3, Bax, $\mathrm{BCl}-2$, and $\mathrm{Bax} / \mathrm{BCl}-2$

Caspase-3, Bax, Bcl-2, and Bax/Bcl-2 expression in Groups B, C, and D was higher than in Group A $(P<0.05-0.01)$. Caspase-3, $\mathrm{Bax}, \mathrm{Bcl}-2$, and $\mathrm{Bax} / \mathrm{Bcl}-2$ expression in Groups
$C$ and $D$ was higher than in Group $B(P<0.05-$ $0.01)$, while $\mathrm{Bcl}-2$ expression was lower than in Group $B(P<0.01$ and $P<0.05)$. Caspase-3, Bax, and $\mathrm{Bax} / \mathrm{Bcl}-2$ expression in Group $D$ was lower than in Group $C(P<0.05-0.01)$, while $\mathrm{Bcl}-2$ expression was higher than in Group C $(P<0.05)$ (Table 2). 
Table 2 - Comparison of expressions of Caspase-3, Bax, Bcl-2, and Bax/Bcl-2 among the groups $(\bar{x} \pm s)$.

\begin{tabular}{llllll} 
Group & $\mathbf{n}$ & Bax & Bcl-2 & Caspase & Bax/Bcl-2 \\
\hline A & 10 & $94.59 \pm 11.30$ & $100.95 \pm 11.98$ & $90.73 \pm 14.03$ & $0.94 \pm 0.12$ \\
B & 10 & $153.32 \pm 17.84^{* *}$ & $142.87 \pm 17.08^{* *}$ & $126.90 \pm 13.78^{* *}$ & $1.07 \pm 0.12^{*}$ \\
C & 10 & $192.80 \pm 27.97^{* * \Delta \Delta}$ & $115.63 \pm 13.95^{* \Delta \Delta}$ & $153.72 \pm 10.79^{* * \Delta \Delta}$ & $1.67 \pm 0.15^{* * \Delta \Delta}$ \\
D & 10 & $172.07 \pm 12.56^{* * \Delta \#}$ & $129.50 \pm 10.55^{* * \Delta \#}$ & $141.97 \pm 11.22^{* * \Delta \#}$ & $1.33 \pm 0.16^{* * \Delta \Delta \#}$ \\
F & - & 51.57 & 17.54 & 47.60 & 55.97 \\
P & - & $<0.01$ & $<0.01$ & $<0.01$ & $<0.01$ \\
Intra-group MS & - & 346.508 & 185.288 & 157.261 & 0.019 \\
\hline
\end{tabular}

Note: $\mathrm{Q}$ test: compared with group $\mathrm{A},{ }^{*} P<0.01$; compared with group $\mathrm{B},{ }^{\Delta} P<0.01$; compared with group $\mathrm{C},{ }^{\sharp} P<0.05$.

\section{- Discussion}

This study used special protective devices to protect the animal airway with instant explosion-produced high-energy shock waves and gas to prepare an ALI model. When an animal's lungs are impacted by shock waves, intra-pulmonary pressure can increase significantly, oppressing the lung tissue and resulting in severe pulmonary contusion, as well as hemorrhage, edema, and exudation in the alveolar cavity and interstitial substances. The $W / D$ ratio will then increase and ventilation/ blood flow ratio becomes disordered; additionally, inflammatory exudation in the lung tissue and apoptosis increase. Compared to Group A, the pathological changes of lung tissue in Group EXP were significant, $\mathrm{PaO}_{2}$ was significantly decreased $(P<0.01)$, and $W / D$ was significantly increased $(P<0.01)$, indicating that the model was successfully prepared. Caspase-3, Bax, and $\mathrm{Bcl}-2$ expression was detected at different time points after successful modeling, and the results showed clear changes in caspase-3, Bax, and Bcl-2 at $4 \mathrm{~h}$ after injury, with the largest differences observed at $12 \mathrm{~h}$ which decreased by $24 \mathrm{~h}$.

Apoptosis is gene-controlled autonomously-programmed death in organisms that stabilizes the internal environment. Studies have shown that apoptosis is involved in the process of $\mathrm{ALI}$, and the following mechanisms have been predicted: first, alveolar epithelial cells undergo apoptosis, decreasing their number, diffusion function decreases, and alveolar effective exchange area decreases, thus affecting the ventilation/blood flow ratio; alternatively, pulmonary capillary endothelial cells occur apoptosis, increasing the permeability of the vascular wall, inflammatory effusion increases, and pulmonary interstitial hemorrhage and edema occur to oppress the alveoli, cause alveolar stenosis, and affect the ventilation/blood flow ratio, leading to hypoxemia; finally, bronchial epithelial cells undergo apoptosis, thus aggravating breathing difficulties ${ }^{14}$. In our studies, early pathological sections revealed a widened pulmonary space, alveolar stenosis, damaged normal alveolar structure, alveolar cavity, and severe edema and exudation in pulmonary interstitial substances, together with inflammatory cell infiltration. The results of TUNEL staining showed that apoptosis occurred early in the experimental group, indicating that apoptosis is involved in the early stage of the occurrence and development of $A L I$, which is consistent with the results of a previous study ${ }^{15}$.

We found that lung tissue apoptosis is affected by the caspase, Bcl-2, Fas/FasL, and other families ${ }^{16}$. Apoptosis induction is achieved by subsequent activation of caspase 
proteins, among which caspase-3 is involved in most apoptotic processes as an apoptotic effector and the most critical factor, caspase-3 lies in a core position of apoptotic activation cascades $^{17,18}$. The results of this study suggest that caspase- 3 is upregulated in the lung tissue of Group EXP, which is consistent with the apoptotic degree of lung cells and results of related studies ${ }^{19}$.

Studies $^{20}$ have shown that the $\mathrm{Bcl}-2$ family is involved in the upstream regulatory mechanism of caspase-3. The expression of $\mathrm{Bcl}-2$ family members plays an important role in the signal transduction of apoptosis. $\mathrm{Bcl}-2$ and $\mathrm{Bax}$ are the most representative apoptosis-inhibitory and pro-apoptosis factors in the $\mathrm{Bcl}-2$ family. Previous studies showed that proapoptotic proteins in the $\mathrm{Bcl}$ 2 family may undergo conformation changes when stimulated by other signals, resulting in apoptosis by affecting the balance of anti- and proapoptotic factors ${ }^{20}$. Bcl-2 is an important anti-apoptotic protein in vivo, and its main anti-apoptotic mechanisms are as follows: (1) inhibiting the activation of apoptosis protease caspases, including caspase-3; (2) direct antioxidant activity; (3) inhibiting the release of proapoptotic substances; and (4) inhibiting proapoptotic protein $\mathrm{Bax}^{21}$. Bax is the most widely distributed proapoptotic protein in the Bcl-2 family ${ }^{22}$. The mechanisms of Bax in promoting apoptosis mainly are as follows: (1) affecting intracellular redox; (2) altering mitochondrial membrane permeability, which affects oxidative phosphorylation and ATP synthesis; (3) activating the caspase signal transduction pathway, thus promoting apoptosis; and (4) antagonizing the antiapoptotic protein $\mathrm{Bcl}-2$. Numerous studies $^{16,20,21}$ have shown that the ratio of Bax/ $\mathrm{Bcl}-2$ is important for apoptosis. The results of this study showed that changes in Bax and the $\mathrm{Bax} / \mathrm{Bcl}-2$ ratio in Group EXP were the same as those observed during apoptosis of lung cells.
Therefore, explosion injury can activate the apoptotic program in the lung tissue of rabbits and increase apoptosis activity while inhibiting antiapoptotic activity, thus inducing apoptosis.

The results of this study showed that the expression of caspase- 3 and Bax was increased, while expression of Bax was reduced, thus affecting the $\mathrm{Bax} / \mathrm{Bcl}-2$ ratio. Therefore, the body may activate caspase- 3 and Bax while inhibiting $\mathrm{Bcl}-2$, thereby promoting apoptosis. However, the results of this study also suggest that apoptotic cells in Group D were lower than in Group C, with decreased caspase-3 and Bax and increased $\mathrm{Bcl}-2$. The possible mechanisms include: (1) late ALI lesions may form under severe conditions, decreasing apoptosis of neutrophils (particularly multinucleated neutrophils, the survival duration of which can affect the degree of damage), thus increasing inflammatory injury ${ }^{23,24}$; $(2)$ in the course of ALI, type II alveolar cells participate in repair with a form of apoptosis, and in late ALI, Bcl-2 shows significant upregulation, while apoptosis of type II alveolar cell decreases, thus decreasing the repair effect and increasing injury ${ }^{25}$; and (3) in late $A L I$, regulatory effects in the body lead to increased anti-apoptotic factor expression. The results of this study show that apoptosis in $A L I$ is first increased and then reduced, consistent with the results of a previous study ${ }^{26}$.

\section{Conclusions}

Plasma caspase-3, Bax, and Bcl-2 show significant changes in the early stage of acute lung injury which are consistent with the degrees of injury and apoptosis. Therefore, detecting the above indices can provide guidance for early diagnosis and intervention of $\mathrm{ALI}$ and effectively assist in the clinical assessment of the severity of lung injury. However, the pathogeneses of ALI are very complex and can be affected by multiple 
influential factors; thus, the mechanisms of apoptosis-induced ALI require further analysis.

\section{References}

1. Singleton JA, Gibb IE, Bull AM, Mahoney PF, Clasper JC. Primary blast lung injury prevalence and fatal injuries from explosions: insights from postmortem computed tomographic analysis of 121 improvised explosive device fatalities. J Trauma Acute Care Surg. 2013;75(2 Suppl 2):S269-74. doi: 10.1097/TA.0b013e318299d93e.

2. Allen TC, Kurdowska A. Interleukin 8 and acute lung injury. Arch Pathol Lab Med. 2014;138(2):266-9. doi: 10.5858/arpa.20130182-RA.

3. Grasso S. Partially assisted ventilationinduced lung injury in early acute respiratory distress syndrome. When real life is different from classical physiology. Am J Respir Crit Care Med. 2017;196(5):538-9. doi: 10.1164/ rccm.201702-0290ED.

4. Andreu G, Boudjedir K, Muller JY, Pouchol E, Ozier Y, Fevre G, Gautreau C, Quaranta JF, Drouet C, Rieux C, Mertes PM, Clavier B, Carlier M, Sandid I. Analysis of transfusionrelated acute lung injury and possible transfusion-related acute lung injury reported to the french hemovigilance network from 2007 to 2013. Transfus Med Rev. 2018;32(1):16-27. doi: 10.1016/j. tmrv.2017.07.001.

5. Lin WC, Chen CW, Huang YW, Chao L, Chao J, Lin YS, Lin CF. Kallistatin protects against sepsis-related acute lung injury via inhibiting inflammation and apoptosis. Sci Rep. 2015;5:12463. doi: 10.1038/srep12463.

6. Tu GW, Shi Y, Zheng YJ, Ju MJ, He HY, Ma GG, Hao GW, Luo Z. Glucocorticoid attenuates acute lung injury through induction of type 2 macrophage. J Transl Med. 2017;15(1):181. doi: 10.1186/s12967-017-1284-7.

7. Jin F, Li C. Seawater-drowning-induced acute lung injury: From molecular mechanisms to potential treatments. Exp Ther Med. 2017;13(6):2591-8. doi: 10.3892/ etm.2017.4302.

8. Zhang Y, Fan L, Xi R, Mao Z, Shi D, Ding D, Zhang $Z$, Wang $X$. Lethal concentration of perfluoroisobutylene induces acute lung injury in mice mediated via cytokine storm, oxidative stress and apoptosis. Inhal Toxicol. 2017;29(6):255-65. doi: 10.1080/08958378.2017.1357772.

9. Komiya K, Akaba T, Kozaki Y, Kadota JI, Rubin BK. A systematic review of diagnostic methods to differentiate acute lung injury/ acute respiratory distress syndrome from cardiogenic pulmonary edema. Crit Care. 2017;21(1):228. doi: 10.1186/s13054-0171809-8.

10.Ware LB, Matthay MA. The acute respiratory distress syndrome. N Engl J Med. 2000;342(18):1334-49. doi: 10.1056/ NEJM200005043421806.

11.Ren M, Wang YM, Zhao J, Zhao J, Zhao ZM, Zhang TF, He J, Ren SP, Peng SQ. Metallothioneins attenuate paraquatinduced acute lung injury in mice through the mechanisms of anti-oxidation and antiapoptosis. Food Chem Toxicol. 2014;73:1407. doi: 10.1016/j.fct.2014.07.039.

12.Fuller BM, Page D, Stephens RJ, Roberts BW, Drewry AM, Ablordeppey E, Mohr NM, Kollef $\mathrm{MH}$. Pulmonary mechanics and mortality in mechanically ventilated patients without acute respiratory distress syndrome: a cohort study. Shock. 2018 Mar;49(3):311-6. doi: 10.1097/SHK.0000000000000977.

13. Matute-Bello G, Martin TR. Science review: apoptosis in acute lung injury. Crit Care. 2003 Oct; 7(5):355-8. doi: 10.1186/cc1861

14.Perl M, Lomas-Neira J, Venet F, Chung CS, Ayala A. Pathogenesis of indirect (secondary) acute lung injury. Expert Rev Respir Med. 2011;5(1):115-26. doi: 10.1586/ers.10.92.

15. Kwon KY, Jang JH, Kwon SY, Cho $\mathrm{CH}$, Oh HK, Kim SP. Cadmium induced acute lung injury and TUNEL expression of apoptosis in respiratory cells. J Korean Med Sci. 2003;18(5):655-62. doi: 10.3346/ jkms.2003.18.5.655.

16.Li CL, Chang L, Guo L, Zhao D, Liu HB, Wang QS, Zhang P, Du WZ, Liu X, Zhang HT, Liu Y, Zhang Y, Xie JH, Ming JG, Cui YQ, Sun Y, Zhang $Z R$, Jiang $C L$. $\beta$-elemene induces caspasedependent apoptosis in human glioma cells in vitro through the upregulation of $\mathrm{Bax}$ and Fas/ FasL and downregulation of Bcl-2. Asian Pac J Cancer Prev. 2014;15(23):1040712. PMID: 25556484.

17. Mcllwain DR, Berger T, Mak TW. Caspase functionsincelldeathand disease. ColdSpring Harb Perspect Biol. 2013;5(4):a008656. doi: 
10.1101/cshperspect.a008656.

18.Damarla M, Parniani AR, Johnston L, Maredia $H$, Serebreni L, Hamdan $O$, Sidhaye VK, Shimoda LA, Myers AC, Crow MT, Schmidt EP, Machamer CE, Gaestel M, Rane MJ, Kolb TM, Kim BS, Damico RL, Hassoun PM. Mitogen-activated protein kinase-activated protein kinase 2 mediates apoptosis during lung vascular permeability by regulating movement of cleaved caspase 3. Am J Respir Cell Mol Biol. 2014;50(5):93241. doi: $10.1165 / \mathrm{rcmb} .2013-03610 \mathrm{C}$.

19.Cross LJ, Matthay MA. Biomarkers in acute lung injury: insights into the pathogenesis of acute lung injury. Crit Care Clin. 2011;27(2):355-77. doi: 10.1016/j. ccc.2010.12.005.

20. Hardwick JM, Soane L. Multiple functions of BCL-2 family proteins. Cold Spring Harb Perspect Biol. 2013;5(2). pii: a008722. doi: 10.1101/cshperspect.a008722.

21.Letai A. Pharmacological manipulation of $\mathrm{Bcl}-2$ family members to control cell death. J Clin Invest. 2005;115(10):2648-55. doi: $10.1172 / \mathrm{JCl} 26250$.

22.Fu $H$, Wang QS, Luo Q, Tan $S$, Su $H$, Tang SL, Zhao ZL, Huang LP. Simvastatin inhibits apoptosis of endothelial cells induced by sepsis through upregulating the expression of $\mathrm{Bcl}-2$ and downregulating Bax. World J Emerg Med. 2014;5(4):291-7. doi: 10.5847/ wjem.j.issn.1920-8642.2014.04.009.

23. Ma HJ, Huang XL, Liu Y, Fan YM. Sulfur dioxide attenuates LPS-induced acute lung injury via enhancing polymorphonuclear neutrophil apoptosis. Acta Pharmacol Sin. 2012;33(8):983-90. doi: 10.1038/ aps.2012.70.

24.Chen G, Xiao B, Chen L, Bai B, Zhang Y, $\mathrm{Xu} Z$, Fu L, Liu Z, Li X, Zhao Y, Liang G. Discovery of new MD2-targeted antiinflammatory compounds for the treatment of sepsis and acute lung injury. Eur J Med Chem. 2017;139:726-40. doi: 10.1016/j. ejmech.2017.08.036.

25.Li W, Qiu X, Jiang H, Han Y, Wei D, Liu J. Downregulation of miR-181a protects mice from LPS-induced acute lung injury by targeting $\mathrm{BCl}-2$. Biomed Pharmacother. 2016;84:1375-82. doi: 10.1016/j. biopha.2016.10.065.

26.Aulakh GK, Suri SS, Singh B. Angiostatin inhibits acute lung injury in a mouse model. Am J Physiol Lung Cell Mol Physiol. 2014;306(1):L58-68. doi: 10.1152/ ajplung.00368.2012.

\section{Correspondence:}

Jian Hao

Department of Cardiopulmonary Rehabilitation Center

128th Hangzhou Hospital, Military Hangzhou

Clinical College, Anhui Medical University

Hangzhou 310007 Zhejiang Province China

Phone: +86 57187341296 / 13857138099

docjianhao@126.com

Received: June 29, 2018

Review: Aug 26, 2018

Accepted: Sept 28, 2018
Conflict of interest: none

Financial source: Key Projects of Medicine and Health in Nanjing Military Area (No. 12MA111). 